\title{
A commentary on "Does the presence of a true radiological rectocele increase the likelihood of symptoms of prolapse?"
}

\author{
Ghazaleh Rostaminia ${ }^{1}$ (i) \\ Received: 6 August 2020 / Accepted: 19 August 2020 / Published online: 25 August 2020 \\ (C) The International Urogynecological Association 2020
}

This retrospective cohort study aimed to investigate whether the presence of a true rectocele on TPUS causes or increases subjective symptoms of prolapse. Women who presented to a tertiary urogynecology unit for symptoms of pelvic floor dysfunction between September 2011-June 2016 were recruited to the study. All subjects underwent an assessment that included a structured interview, a clinical (POP-Q) examination and a 4D transperineal ultrasound scan. A physician-administered questionnaire queried for symptoms of prolapse. The subjective bother of prolapse symptoms was quantified using a visual analogue scale. Offline analysis of TLUS data was performed by assessors blinded to symptoms and clinical findings.

Data of 348 women, with rectocele-dominant descent on clinical examination, that is, with $\mathrm{Bp}$ being the most distal point on POP-Q examination, entered the analysis. Mean patient age was 60 (33-86) years with a mean BMI of $31(18-$ $55) \mathrm{kg} / \mathrm{m}^{2}$. One hundred fifty-three $(44 \%)$ patients presented with prolapse symptoms (defined as a "lump" or a "bulge" in the vagina) at an average bother of $3 / 10(0-10)$ on a visual analogue scale. Significant posterior compartment prolapse was present in $331 / 348(95 \%)$. Of these, 272 were true rectoceles on TPUS, with a pocket depth $\geq 10 \mathrm{~mm}$. There were 35 women (11\%) with only perineal hypermobility (that is, descent of the rectal ampulla without a true rectocele or enterocele), and in 24 women (7\%) an isolated enterocele was found. Thirty-one (9\%) had a clinically significant cystocele and $17(6 \%)$ had a significant uterine prolapse.

Ghazaleh Rostaminia

ghazalerostaminia@yahoo.com

1 NorthShore University HealthSystem, Skokie, IL, USA
Clinical rectocele (Bp on POP-Q) and true rectocele on TPUS were found to significantly increase the likelihood of prolapse symptoms. Bp on POP-Q and true rectocele on TPUS were both significantly associated with prolapse symptoms; however, on multivariate analysis the latter became nonsignificant.

In summary, this study suggested that the presence of a true rectocele as diagnosed on translabial ultrasound imaging does not contribute substantially to the clinical symptom of a "lump" or "bulge" experienced by patients. Symptoms of posterior compartment prolapse seem to be due to descent of the posterior vaginal wall, regardless of underlying anatomical causes. The current study has only focused on the symptom of "bulge" related to the posterior vaginal prolapse and showed that performing ultrasound and evaluating rectal shape and motion do not improve the clinical assessment related to it. However, when it comes to the functional aspects of pelvic organ prolapse, which include symptoms such as fecal incontinence or obstructed defecation, it would have been interesting to see whether the same outcome would have been seen. Clinicians who are interested in implementing pelvic floor ultrasound in their practices should be aware of these two distinctive categories of prolapse-related symptoms and the role that imaging will play in their assessments.

\section{Compliance with ethical standards}

Conflicts of interest None.

Publisher's note Springer Nature remains neutral with regard to jurisdictional claims in published maps and institutional affiliations. 